\title{
50 Jahre Römische Verträge - Vorreiter einer modernen Rechtsentwicklung
}

\author{
Stephan Hobe und Andrea End*
}

Die Unterzeichnung der Römischen Verträge am 25. März 1957 zur Gründung der EURATOM und der Europäischen Wirtschaftsgemeinschaft (EWG) gibt Anlass, die Geschichte der Integration auf den Prüfstand zu stellen. Immerhin sind 50 Jahre ein nicht unbedeutendes Stück Weg, welches die Staaten Europas in unterschiedlicher Zusammensetzung gemeinsam gegangen sind. Aufbauend auf dem visionären Konzept des Schuman-Plans im Jahre 1950 setzen die Römischen Verträge die Erfolgsgeschichte der friedlichen Zusammenarbeit europäischer Länder nicht nur fort. Sie bilden nach dem Modell des Vertrages zur Gründung der Europäischen Gemeinschaft für Kohle und Stahl (EGKS) einen ersten, bis heute grundlegenden Meilenstein staatsübergreifender und integrativer Zusammenarbeit, indem sie in einzigartiger Weise dem Bedingungszusammenhang zwischen wirtschaftlichen Interessen einerseits und der Sicherung von Frieden und Wohlstand andererseits in Europa Rechnung tragen. Die Grundidee des Schuman-Plans, nämlich die Fusion von staatlichen Souveränitätsrechten, hat in den letzten 50 Jahren nicht nur der Teilung Europas und dem Kalten Krieg standgehalten. Darüber hinaus hat sie nach dem Beitritt Spaniens, Portugals und Griechenlands zur Überwindung der Folgen diktatorischer Regime in Südeuropa beigetragen. Schließlich hat sich die gesamteuropäische Ausrichtung dieser den Verträgen zu Grunde gelegten Idee mit der Aufnahme der osteuropäischen Staaten im Jahre 2004 sowie Anfang des Jahres 2007 bestätigt, die das bewusste Ende und die Abkehr von der Teilung Europas durch die Trennung in unterschiedliche ideologische Blöcke markiert. Nach dem vorläufigen Scheitern des Verfassungsvertrages in Frankreich und den Niederlanden muss aktuell die Frage aufgeworfen werden, ob mit diesem Vertragswerk ein den Römischen Verträgen ähnlich bedeutender Schritt noch getan werden kann.

Bereits zum Zeitpunkt der Unterzeichnung der Römischen Verträge stand fest, dass es die fortlaufende Verwirklichung der Vision der europäischen Gründerväter sein sollte, die, nun nicht mehr sektoral auf die Kohle- und Stahlindustrie begrenzt, Europa immer wieder neu entstehen lassen sollte. Im Mittelpunkt der nachfolgenden Betrachtung sollen daher die mit den Römischen Verträgen initiierten Prozesse stehen. Diese lassen sich aus einer juristischen Perspektive insbesondere anhand des Begriffs der Integration, des Begriffs der Supranationalität und der Entwicklung von Verfassungsstrukturen über den staatlichen Bereich hinaus darstellen. Ohne Anspruch auf Vollständigkeit sollen die von den Verfassern ausgewählten Gesichtspunkte ein umfassendes Bild zurückliegender Entwicklungen und ihrer rechtlichen Bezüge entwerfen.

\section{Die Reichweite des Konzepts der europäischen Integration}

Ein erstes signifikantes Faktum ist die tatsächliche Überzeugungskraft der europäischen Integration, ${ }^{1}$ die inzwischen nicht nur die sechs Gründungsmitgliedstaaten (mit rund 200

* Prof. Dr. Stephan Hobe, LL.M. (McGill), Inhaber des Lehrstuhls für Völkerrecht, Europarecht, europäisches und internationales Wirtschaftsrecht, Direktor des Instituts für Luft- und Weltraumrecht, Universität zu Köln.

Andrea End, wissenschaftliche Mitarbeiterin am Lehrstuhl für Völkerrecht, Europarecht, europäisches und internationales Wirtschaftsrecht, Universität zu Köln.

1 Zum Begriff der Integration siehe Hermann Mosler: Die Entstehung des Modells supranationaler und gewaltenteilender Staatenverbindungen in den Verhandlungen über den Schuman-Plan, in: Ernst von Caemmerer/ Hans-Jürgen Schlochauer/Ernst Steindorff (Hrsg.): Probleme des Europäischen Rechts, Festschrift für Walter Hallstein zu seinem 65. Geburtstag, Frankfurt a. M. 1966, S. 355-386, hier S. 371-372. 
Millionen Bürgern) der Römischen Verträge, sondern nunmehr 27 Mitgliedstaaten (mit über 490 Millionen Bürgern) der Europäischen Gemeinschaft beziehungsweise Europäischen Union dazu bewegt hat, Hoheitsrechte auf europäische Institutionen zu übertragen. Die Gründung der EWG und der EURATOM 1957 war nicht der erste Schritt in diese Richtung, ${ }^{2}$ denn die Montanunion war ja bereits drei Jahre vorher gegründet worden; der Vertrag, der für eine Dauer von 50 Jahren geschlossen wurde, lief im Juli 2002 aus. Die Gründung der EURATOM setzt aber den Ansatz, der zur Entstehung der Montanunion geführt hatte, fort. Es geht erneut um die sektorale Vergemeinschaftung der Kontrollbefugnisse über rüstungserhebliche Güter, hier die Frage des Zugriffs auf die friedliche Nutzung der Kernenergie.

Von ungleich größerer Bedeutung war allerdings die Gründung der Europäischen Wirtschaftsgemeinschaft, die den sektoralen Ansatz überwinden und zugleich nach dem Vorbild der Montanunion ein neues, großes europäisches Marktgebiet unter den zunächst sechs Mitgliedstaaten schaffen sollte. ${ }^{3}$ Der gemeinsame Markt sollte insbesondere mit der Einrichtung einer Zollunion unter Abschaffung der Binnenzölle, der Einführung eines gemeinsamen Außenzolls ${ }^{4}$, der rechtlichen Gewährleistung der fünf Grundfreiheiten (Warenverkehrsfreiheit, Arbeitnehmerfreizügigkeit und Niederlassungsfreiheit, Dienstleistungsfreiheit und schließlich Kapitalverkehrsfreiheit) ${ }^{5}$, der Angleichung der Rechts- und Verwaltungsvorschriften ${ }^{6}$ sowie weiteren wirtschaftsrechtlichen Regelungen ${ }^{7}$ entstehen. Das die Grundfreiheiten prägende Diskriminierungsverbot ${ }^{8}$ - das Verbot der unterschiedlichen Behandlung auf Grund der Staatsangehörigkeit - wurde in dieser Phase der wirtschaftlichen Vereinigung Europas zum maßgeblichen Leitgedanken. Die Bedeutung der Staatsangehörigkeit als Differenzierungskriterium wurde nivelliert. Die mittels der genannten Rechte und Rechtsinstrumente sektorübergreifende Öffnung der Staatsgrenzen war das eigentliche Novum des EWG-Vertrages. Nach dem Modell des EGKS-Vertrages wurden die vier Institutionen der Montanunion - die Hohe Behörde als Kommission, der Ministerrat, die parlamentarische Versammlung und der Gerichtshof - etabliert. ${ }^{9}$ Die Rechtssetzungsbefugnisse verblieben damit bei der Kommission und dem Ministerrat, der in der EWG jedoch ein stärkeres Gewicht erhielt. ${ }^{10}$ Die Aufgabenbereiche aller Institutionen wurden auf den gesamten wirtschaftlichen Bereich ausgeweitet. Die rechtlich neuartige Dimension, die so mit dem EWG-Vertrag angelegt und in den folgenden 50 Jahren mit Leben erfüllt worden ist, hat der erste Präsident der Kommission, Walter Hallstein, prägend folgendermaßen zusammengefasst: Die Europäische Wirtschaftsgemeinschaft ,ist Schöpfung des Rechts, sie ist Rechtsquelle und sie ist Rechtsordnung." ${ }^{11}$ Das ist der eigentliche Gehalt der rechtlichen Integration.

Man wird ohne Zögern sagen können, dass die zunächst auf die Schaffung eines gemeinsamen Marktes zielende Integration die maßgebliche Triebfeder sein sollte, um über die wirtschaftliche auch die politische Integration zu erreichen. ${ }^{12}$ Für die wirtschaftspolitischen

2 Franz Knipping: Rom, 25. März 1957, Die Einigung Europas, München 2004, S. 98-102.

3 Erster Teil, Artikel 2 des EWG-Vertrages; siehe dazu Walter Hallstein: Der unvollendete Bundesstaat - Europäische Erfahrungen und Erkenntnisse, Düsseldorf 1969, S. 19-29.

4 Erster Teil, Artikel 3 lit. a, b sowie Zweiter Teil, Titel I, Kapitel 1 des EWG-Vertrages.

5 Erster Teil, Artikel 3 lit. c sowie Zweiter Teil, Titel I, Kapitel 2 sowie Titel III des EWG-Vertrages.

6 Erster Teil, Artikel 3 lit. h sowie Dritter Teil, Titel I, Kapitel 3 des EWG-Vertrages.

7 Erster Teil, Artikel 3 lit. f. sowie Dritter Teil, Titel I, Kapitel 1 des EWG-Vertrages.

8 Erster Teil, Artikel 7 des EWG-Vertrages.

9 Erster Teil, Artikel 4 sowie Fünfter Teil, Titel I Kapitel 1 des EWG-Vertrages.

10 Mosler: Die Entstehung des Modells supranationaler und gewaltenteilender Staatenverbindungen, hier S. 359360.

11 Hallstein: Der unvollendete Bundesstaat, S. 33 und insgesamt S. 33-36.

12 Siehe Hallstein: Der unvollendete Bundesstaat, S. 18-29, 93-98; Knipping: Rom, 25. März 1957, S. 102. 
Bereiche der EWG und der EURATOM ist dies weitgehend gelungen. Beispielhaft sei auf die personelle Beziehung der EWG zu den Bürgern der Gemeinschaft verwiesen. Seit der anfänglich rein wirtschaftlichen und eher passiven Stellung des sogenannten Marktbürgers, ${ }^{13}$ die insbesondere durch die Verbraucherschutzrechte aktiviert wurde, ${ }^{14}$ ist inzwischen der status activus des europäischen Bürgers vor allem im Vertrag von Maastricht mit der Schaffung der Unionsbürgerschaft deutlich gestärkt worden. Die mittels der Unionsbürgerschaft verliehenen politischen Mitwirkungsrechte integrieren die Bürger nun staatsübergreifend, was mit der signifikanten, wenngleich bisher relativ selten genutzten Möglichkeit des kommunalen Ausländerwahlrechts für EU-Bürger an deren Wohnort zum Ausdruck kommt. ${ }^{15}$

Außerhalb des wirtschaftspolitischen Bereichs ist die Entwicklung freilich nicht so gradlinig verlaufen. Es zeigt sich, dass die kurz nach der Gründung der Montanunion als zweites europäisches politisches Projekt gedachte Schaffung der europäischen Verteidigungsgemeinschaft (EVG) und deren Scheitern in der französischen Nationalversammlung weitreichende Folgen nach sich ziehen sollte. ${ }^{16}$ Diese Folgen sind vor allem an der verzögerten politischen Integration Europas, besonders im außen- und verteidigungspolitischen Bereich, ablesbar. So sollte es bis zur Etablierung der Europäischen Union dauern, dass basierend auf den ersten Reformschritten der Einheitlichen Europäischen Akte zur Vollendung des Binnenmarktes, ein Versuch gemacht wurde, durch die neue Drei-Säulenstruktur die Integration im Bereich der Gemeinsamen Außen- und Sicherheitspolitik (GASP) und im Bereich der polizeilich und justiziellen Zusammenarbeit in Strafsachen (PJZS) voranzutreiben. ${ }^{17}$ Jedoch drücken in aller Regel bereits die Normativakte der intergouvernementalen Zusammenarbeit in diesen Bereichen, wie etwa der gemeinsame Standpunkt gemäß Artikel 15 des EU-Vertrages, die Möglichkeit der Geltendmachung staatlicher Vorbehalte zur Identitätswahrung trefflich aus. ${ }^{18}$ Dies und das Fehlen der Freiheitsrechte sowie der weiteren Rechtsinstrumente, die im Rahmen der Gemeinschaften als erste Säule den Prozess der Integration am Laufen halten, sind unter anderem für die so zögerlich voranschreitende politische Integration mitverantwortlich. Die gleichlaufenden wirtschaftlichen Interessen waren Voraussetzung für die Etablierung einer rechtlichen Kooperation und des materiellen Rechtskorpus der Gemeinschaften; diese Voraussetzung ist zumindest im Bereich der GASP als zweite Säule nicht gegeben. Ein Beispiel dafür gaben die so unterschiedlichen Auffassungen der ständigen Mitglieder des Sicherheitsrates der Vereinten Nationen, Frankreich und Großbritannien, während des letzten Irak-Krieges.

Innerhalb der PJZS, der dritten Säule, ist indes die Reichweite der Kooperation durchaus anders zu beurteilen. Beweis dafür ist die Überführung von immer mehr Bereichen der PJZS in die $\mathrm{E}(\mathrm{W}) \mathrm{G}$, so etwa die gemeinsame Asylpolitik und wohl, trotz gewisser Schwierigkeiten, perspektivisch etwa der Bereich der polizeilichen Verfolgung. ${ }^{19}$ Auch wurde jüngst mit

13 Hans Peter Ipsen: Europäisches Gemeinschaftsrecht, Tübingen 1972, S. 742-751; Norbert Reich: Europäisches Verbraucherrecht, Baden-Baden 1996, S. 27-30.

14 Siehe dazu das erste und zweite Programm des Rates für eine Politik zum Schutz und zur Unterrichtung der Verbraucher, Amtsblatt C 92, 25.04.1975, S. 2 und Amtsblatt C 133, 03.06.1981, S. 1, sowie das Grünbuch der Kommission: Zugang der Verbraucher zum Recht, KOM(93) 576 endgültig, 16.11.1993.

15 Stephan Hobe: Die Unionsbürgerschaft nach dem Vertrag von Maastricht, in: Der Staat 3/1993, S. 245-268; in Bezug auf die Europäische Grundrechte-Charta siehe Stephan Hobe: Bürgerrechte, in: Peter Tettinger/Klaus Stern: Europäische Grundrechte-Charta, München 2006, S. 644-661.

16 Gerhard Brunn: Die Europäische Einigung von 1945 bis heute, Stuttgart 2002, S. 88-99; Jürgen Elvert: Die europäische Integration, Darmstadt 2006, S. 57-62.

17 Siehe zum Vertrag von Maastricht Peter Huber: Maastricht - ein Staatsstreich?, Stuttgart u.a. 1993.

18 Siehe etwa Stephan Hobe: Europarecht, Köln 2006, S. 315-337; Stephan Hobe: Der offene Verfassungsstaat zwischen Souveränität und Interdependenz, Berlin 1998, S. 331-334.

19 Hobe: Europarecht, S. 327-328. 
dem „Pupino“-Urteil vom EuGH die Frage aufgeworfen, inwieweit eine Annäherung der PJZS an das Integrationsmodell der EWG erfolgen kann. ${ }^{20}$

Grundsätzlich bleibt seit dem Scheitern der EVG die Schwierigkeit des Zusammenwirkens der europäischen Staaten in nicht rein wirtschaftlich geprägten Politikbereichen bestehen, wodurch gleichzeitig eine gewisse Grenze der Reichweite des Konzepts der Integration gezogen wird.

Nicht verkannt werden darf schließlich, dass diese Reichweite durch die ständig steigende Zahl der Mitgliedstaaten mitbestimmt wird, indem mittlerweile nicht nur sechs sondern 27 Mitgliedstaaten nicht nur rechtlich unterschiedliche Ansprüche an die Integration stellen. Andererseits kann nichts trefflicher die Tatsache zum Ausdruck bringen, dass das Grundkonzept der EWG und der EURATOM von vielen Staaten getragen wird, also das gemeinschaftliche Europa eben als ein Hort der Stabilität, der Sicherheit und des Fortschritts angesehen wird.

\section{Das Voranschreiten der ökonomischen Integration}

Die Basis für die wirtschaftliche Integration war also von Anfang an durch die Römischen Verträge gelegt. Das Vorhaben der Gründer des EWG-Vertrages ,die Grundlage für einen immer engeren Zusammenschluss der europäischen Völker zu schaffen“ ${ }^{\text {21 }}$ hat sich zudem durch weitere bedeutende ökonomische Schritte realisiert. Ausgehend von dem Grundgedanken des Schuman-Plans, durch die Fusion von Hoheitsrechten auf europäischer Ebene hinsichtlich des Kohle- und Stahlsektors einen Krieg undenkbar und auch materiell unmöglich zu machen, hat sich die Idee der Integration für den gesamten ökonomischen Bereich zu einer Form der Dependenz der Staaten innerhalb der E(W)G entwickelt. Dies gilt zum einen für die recht weitreichende Schaffung eines gemeinsamen Marktes, wo das Gemeinschaftsrecht die nationalen Rechtsordnungen weitgehend überlagert. Ein deutlicher Schritt war hierfür die Rechtsprechung des EuGH, die die häufig dem Vertrag nach nur als Diskriminierungsverbote etablierten Grundfreiheiten hin zu Beschränkungsverboten ausgelegt hat. ${ }^{22}$ Zum anderen gilt dies für die bereits mit dem EWG-Vertrag beabsichtigte etappenweise Schaffung der Währungsunion. ${ }^{23}$ Denn die durch den Wegfall der flexiblen Wechselkursmechanismen und die Schaffung einer allgemeinen europäischen Währung hervorgerufene Abhängigkeit nationalstaatlicher Wirtschafts-, Arbeitsmarkt- und Finanzpolitik kettet das Schicksal der Staaten äußerst eng aneinander. Dies gilt jedenfalls für solche Staaten, die die Kriterien für die Aufnahme in die Währungsunion, die heute immerhin 13 Mitgliedstaaten der Union umfasst, erfüllen und dieser auch beitreten wollen. ${ }^{24}$ Ergebnis dieses Prozesses ist, dass es in immer höherem Maße neben dem nationalstaatlichen auch ein europäisches Interesse an der jeweils guten wirtschaftlichen Verfassung der Staaten Europas gibt. Perspektivisch vermag man sich die Umkehrbarkeit der Etablierung des Integrationsgedankens kaum mehr vorzustellen. Insofern mag die eigentlich nur im rechtswissenschaftlichen Schrifttum $^{25}$ eingehend erörterte Frage eines möglichen freiwilligen Austritts eines Mit-

20 EuGH Rs. C-105/03, Slg. 2005, S. I-5285; siehe dazu Stephan Hobe: Zu den Wirkungen von EU-Rahmenbeschlüssen im mitgliedstaatlichen Recht, in: Juristische Ausbildung 2006, S. 859-862.

21 Präambel des EWG-Vetrages.

22 So zum Beispiel für die Arbeitnehmerfreizügigkeit EuGH Rs. C-415/93, Slg. 1995, S. I-4921 „Bosman“; siehe dazu Stephan Hobe/Christian Tietje: Europäische Grundrechte auch für Profi-Sportler, in: Juristische Schulung 1996, S. 486-493; Martin Nettesheim: Die europarechtlichen Grundrechte auf wirtschaftliche Mobilität (Art.48, 52 EGV), in: Neue Zeitschrift für Verwaltungsrecht 1996, S. 342-345.

23 Dritter Teil, Titel II, Kapitel 1 und 2 des EWG-Vertrages.

24 Elvert: Die europäische Integration, S. 93-101; bemerkenswert ist, dass 2007 mit Slowenien der erste Staat des ehemaligen Ostblocks in die Währungsunion nach Erfüllung der Kriterien aufgenommen werden konnte.

25 Hobe: Europarecht, S. 35-36; Huber: Maastricht - ein Staatsstreich?, S. 33-34. 
gliedstaates heute aus der Europäischen Gemeinschaft und der Europäischen Union ad absurdum geführt werden, denn in der Realität scheint ein solcher Austritt doch eher unwahrscheinlich zu sein. Es ging bei der Einführung dieser Option in Art. I-60 des Verfassungsvertrages auch in erster Linie wohl darum, keinen Freibrief zum Austritt auszustellen, sondern für einen solchen Austritt ein möglichst geordnetes Verfahren zu schaffen.

Diese Form der Dependenz lässt daher die gemeinsame „Grundlage für einen immer engeren Zusammenschluss der europäischen Völker" entstehen. In der Präambel des EWGVertrags angelegt - ,durch diesen Zusammenschluss ihrer Wirtschaftskräfte Frieden und Freiheit zu wahren und zu festigen“ - finden diese Werte heute in Artikel 6 des EU-Vertrages ihren Ausdruck. Dies hatte letztlich die Beschränkung der Gemeinschaften auf die liberalen Marktwirtschaften Westeuropas zur Folge. Um gemäß der Präambel „Frieden und Freiheit zu wahren“ bedarf es zusätzlich der in Artikel 6 des EU-Vertrages genannten Achtung der Menschenrechte und Grundfreiheiten, Demokratie und Rechtsstaatlichkeit - sie bilden sozusagen die Basis des acquis communautaire.

\section{Das Modell der Supranationalität}

Die Überwachung, Sicherstellung sowie die Umsetzung der einzelnen Integrationsschritte obliegt nach dem EWG- und EURATOM-Vertrag der Kommission. ${ }^{26}$ Die Kommission hatte ihr Vorbild in der 1953 etablierten Hohen Behörde der Montanunion, die ihrerseits das neue rechtliche Konstrukt der Supranationalität als Handlungsinstrument eingeführt hat. Anders als bei ,normalen“ internationalen Organisationen sollte es diesem Organ, der neu zu schaffenden europäischen Organisation, auch in den Gemeinschaften möglich sein, nicht nur direkt auf die Mitgliedstaaten einzuwirken, sondern auch direkte Durchgriffsrechte auf deren Bürgerinnen und Bürger zu erhalten. Von der ursprünglichen Begriffsdefinition der institutionellen ,Supranationalität' der Hohen Behörde ausgehend, hat sich durch die Gewichtsverlagerung im Rechtssetzungsverfahren auf den Ministerrat in der EWG der Begriff auch inhaltlich verändert. ${ }^{27}$ Über die staatsübergreifende Institutionalisierung hinaus beinhaltet er nun auch die für die Integration wesentliche Übertragung und Fusionierung von Hoheitsrechten und damit die Frage nach der Kompetenz zum Handeln.

Entsprechende Akte sollten also Durchgriffswirkung bis zum Einzelnen haben, und der Einzelne sollte sich auf diese entsprechenden Akte berufen können. Dieses Modell wird flankiert von der Sekundärrechtssetzungsmöglichkeit der Gemeinschaften aufgrund der jeweiligen kompetenzrechtlichen Ermächtigungsgrundlage und hat hier seinen deutlichsten Ausdruck. Diese gleichsam ständige Rechtsfortbildung durch direkt wirkende beziehungsweise im Grundsatz nicht anzuzweifelnde und nur im Ausmaß anzupassende Sekundärrechtssetzung ist es, die die Integration tagtäglich lebendig hält. Wesentliche Ereignisse, wie etwa die Möglichkeit, sich auch auf Richtlinien und Verordnungen als Marktbürger berufen zu können, haben zu dieser Erfahrbarkeit Europas für den Marktbürger beigetragen. Sie haben in einer unübersehbaren Weise einen Anpassungsdruck des nationalen Rechts an die gemeinschaftlichen Vorgaben erzeugt.

Der Europäische Gerichtshof (EuGH) hat in seiner berühmten „effet utile“-Rechtsprechung, ${ }^{28}$ die sich als überaus gemeinschaftsfreundlich erwiesen hat, ebenfalls versucht, für

26 Erster Teil, Artikel 4 Nr. 1 sowie Dritter Teil, Kapitel 1, Abschnitt III des EWG-Vertrages; Erster Teil, Artikel 3 Nr. 1 sowie Dritter Teil, Kapitel 1, Abschnitt III des EURATOM-Vertrages.

27 Zum Begriff der Supranationalität siehe Mosler: Die Entstehung des Modells supranationaler und gewaltenteilender Staatenverbindungen, S. 367.

28 So zum Beispiel EuGH Rs. 106/77, Slg. 1978, S. 629, „Simmenthal II“. 
europäisches Recht eine möglichst hohe Durchsetzungschance zu eröffnen. Ihm entstammt das Postulat unmittelbarer Anwendbarkeit des Europarechts, ${ }^{29}$ der direkten Berufungsmöglichkeit der Marktbürger auf eben jenes Recht, ${ }^{30}$ die Bezeichnung des europäischen Rechts als Rechtsordnung eigener Art zwischen Völkerrecht und nationalem Recht ${ }^{31}$ und schließlich die Indienstnahme des Marktbürgers für die Disziplinierung der Staaten, zum einen durch die Möglichkeit der Berufung auf noch nicht implementierte Richtlinien ${ }^{32}$ und zum anderen durch die Rechtsprechung zur Staatshaftung der Mitgliedstaaten bei Umsetzungsfehlern jedweder $\mathrm{Art}^{33}$.

Betrachtet man diese weitreichenden rechtlichen und tatsächlichen Konsequenzen, stellt sich nicht zuletzt unter dem Aspekt der Übertragung von Hoheitsgewalt von den Mitgliedstaaten auf die Gemeinschaften die Frage nach der Legitimation dieser supranationalen Integration seit den Römischen Verträgen. ${ }^{34}$ Herrschaftsausübung in Europa wird einerseits über die Mitgliedstaaten legitimiert, was sinngemäß in der durch den EWG-Vertrag gestärkten Position des Rates zum Ausdruck kommt. Andererseits versucht das Parlament etwa bei der Rechtssetzung zunehmend die Begründung eines eigenständigen Legitimationsstranges.

\section{Die Frage der Grundrechte}

Die zunächst untergeordnete Bedeutung, die den europäischen Völkern und Bürgern in den Römischen Verträgen zugekommen war, hat zu einer juristisch gesehen kritischen Lücke vor allem im EWG-Vertrag geführt. Dies war die Frage der Geltung der Grundrechte im Gemeinschaftsrecht, sieht man einmal von dem in diese Richtung weisenden grundrechtsspezifischen Diskriminierungsschutz ab. Kritisch war diese Lücke, weil es doch im Gemeinschaftsrecht als Konsequenz der Supranationalität um die Ausübung genuin eigener Hoheitsgewalt durch die Gemeinschaften geht. Insofern gibt es dann , grundrechtstypische Gefährdungslagen', wenn ein Gemeinschaftsbürger oder eine Gemeinschaftsbürgerin von einem europäischen Hoheitsakt in seinen/ihren Grundrechten betroffen ist. Und bekanntlich war es im Wesentlichen die Rechtsprechung des EuGH, die folgerichtig seit dem berühmten Urteil in der Rechtssache „Stauder“35 sukzessiv den Grundrechtsschutz im Gemeinschaftsrecht etabliert hat. Damit hat der EuGH auch seine in Artikel 164 des EWG-Vertrages zugedachte objektive Rolle ,zur Wahrung des Rechts bei der Auslegung und Anwendung dieses Vertrags" unter Beweis gestellt.

Der EuGH judizierte im Einzelnen, dass die Grundrechtsverbürgungen in den Mitgliedstaaten, besonders versinnbildlicht auch anhand eines gewissen Realisierungsniveaus in der Europäischen Menschenrechtskonvention, ${ }^{36}$ für das Vorhandensein allgemeiner Grundsätze

29 Grundlegend EuGH Rs. 26/62, Slg. 1963, S. 1, „van Gend \& Loos“.

30 EuGH Rs. 57/65, Slg. 1966, S. 257, „Alfons Lütticke“.

31 EuGH Rs. 6/64, Slg. 1964, S. 1251, „Costa/ENEL“; Rs. 11/70, Slg. 1970, S. 1125, „Internationale Handelsgesellschaft".

32 Seit EuGH Rs. 9/70, Slg. 1970, S. 825, „Leberpfennig“.

33 EuGH verb. Rs. C-6/90 u. C-9/90, Slg. 1991, I S. 5357, „Francovich“; verb. Rs. C 46/93 u. C-48/93, Slg. 1996, I S. 1029, „Factortame III“; Rs. C-5/94, Slg. 1996, I S. 2553, „Hedley Lomas“; Rs. C-224/01, Slg. 2003, I S. 10239, „Köbler“.

34 Siehe allgemein zu diesem Aspekt Hermann Mosler: Die Erweiterung des Kreises der Völkerrechtssubjekte, in: Zeitschrift für ausländisches öffentliches Recht und Völkerrecht 1962, S. 1-48, hier S. 33; speziell zu den Defiziten des Nationalstaatensystems zur Zeit der Römischen Vertrage Wilfried Loth: Der Weg nach Rom Entstehung und Bedeutung der Römischen Verträge, in: integration 1/2007, S. 36-43, hier S. 36-38.

35 EuGH Rs. 29/69, Slg. 1969, S. 419.

36 Siehe dazu Dieter Kraus: Grundrechtsschutz in der Europäischen Union, in: Rainer Grote/Thilo Marauhn (Hrsg.): EMRK/GG - Konkordanzkommentar zum europäischen und deutschen Grundrechtsschutz, Tübingen 2006, S. 97-154. 
des Gemeinschaftsrechts sprächen, die einen solchen Grundrechtsschutz anerkennen. Der dogmatische Schluss des EuGH legt das Ineinandergreifen der Gemeinschaftsrechtsordnung mit den nationalen Rechtsordnungen nahe. Hier stellt sich insbesondere in der Frontstellung nationalen Verfassungsrechts mit europäischem Sekundärrecht und der verdrängenden Wirkung des letzteren manch grundsätzliche Frage, die noch nicht gelöst ist. So fehlt es an der Ausarbeitung einer europäischen Wesentlichkeitstheorie, ${ }^{37}$ in der die Fundamente rechtsstaatlichen Gebarens festgelegt sind. Dagegen ist die Frage nach dem Verhältnis der deutschen Grundrechte zum Gemeinschaftsrecht infolge der "Solange“-Rechtsprechung des Bundesverfassungsgerichts als gelöst anzusehen. ${ }^{38}$ Jedenfalls hat sich der Gedanke des Grundrechtsschutzes durchgesetzt, obwohl praktisch keine Urteile des EuGH bekannt sind, in denen Gemeinschaftsbürger wegen Grundrechtsverletzungen Recht gegeben wurde. ${ }^{39}$ Auf dem Europäischen Gipfel in Köln im Jahr 1999 war man nun der einhelligen Auffassung, die bisher nicht verschriftlichte, der Rechtsprechung des EuGH entsprechende Verbürgung der Grundrechte als allgemeine Rechtsgrundsätze bedürfe der Kodifikation. Das hat schließlich zur - allerdings bislang rechtlich unverbindlichen ${ }^{40}$ - feierlichen Erklärung der Charta der Grundrechte geführt. ${ }^{41}$ Eben jene Grundrechte-Charta soll auch Teil der Europäischen Verfassung werden.

\section{Der Konstitutionalisierungsprozess}

Die Entwicklung der Gemeinschaftsgrundrechte ist ein Teil des mit den Römischen Verträgen begonnenen Konstitutionalisierungsprozesses. ${ }^{42}$ Löst man sich von nationalstaatlichen Vorstellungen der Verfassung, so ist es nicht nur legitim, sondern ausgesprochen angemessen, für die Gemeinschaften das Bestehen einer Verfassung anzunehmen. ${ }^{43}$ Bereits bei der Gründung der Montanunion wurde überlegt, den Begriff ,Constitution“ in den Titel des Vertrages mit aufzunehmen. ${ }^{44}$ Auch ist vom EuGH zwar mehr behauptet als begründet worden, dass der EWG-Vertrag die „Verfassungsurkunde der Gemeinschaft“ sei, ${ }^{45}$ doch ist der

37 Dazu neuerdings Sonja Röder: Der Gesetzesvorbehalt der Charta der Grundrechte der Union im Lichte einer europäischen Wesentlichkeitstheorie, erscheint voraussichtlich im Laufe des Jahres 2007.

38 BVerfGE 37, 271, „Solange I“; 73, 339, „Solange II“; 89, 155 „Maastricht“; 102, 147 „Bananenmarkt“.

39 Kritisch sehen dies Jason Coppel/Aidan O’Neill: The European Court of Justice: Taking Rights Seriously?, in: Common Market Law Review 2/1992, S. 669-692; zu Recht wohl dagegen Joseph Weiler/Nicholas Lockhart: „Taking Rights Seriously“ Seriously, in: Common Market Law Review 32 (1995), S. 51-94 (Teil I), S. 597627 (Teil II)

40 Siehe dazu aber jüngst EuGH Rs. C-540/03, Europäische Zeitschrift für Wirtschaftsrecht 2006, S. 566-573 mit Anm. von Michael Fremuth.

41 Hobe: Europarecht, S. 158-159; Peter Michael Mombaur: Entstehungsgeschichte der Charta der Grundrechte der Union, in: Peter Tettinger/Klaus Stern (Hrsg.): Kölner Gemeinschaftskommentar zur Europäischen Grundrechte-Charta, München 2006, S. 213-220.

42 Stephan Hobe: Bedingungen, Verfahren und Chancen europäischer Verfassunggebung, in: Europarecht 1/ 2003, S. 1-16, hier S. 4; Anne Peters: Elemente einer Theorie der Verfassung Europas, Berlin 2001, S. 376; Sonja Puntscher Riekmann/Wolfgang Wessels: European Constitutionalism at the Crossroads, in: Anne Peters: The Making of a European Constitution, Wiesbaden 2006, S. 7-31, hier S. 24-25.

43 Christoph Dorau: Die Verfassungsfrage der Europäischen Union, Baden-Baden 2001, S. 158-159; Hobe: Bedingungen, Verfahren und Chancen europäischer Verfassunggebung, S. 1-16; Peters: Elemente einer Theorie der Verfassung Europas, S. 91-92. Siehe dazu auch mit aktuellen Bezügen in polnischer Sprache Stephan Hobe: Niemiecka Prezydencja przyszłść Traktatu konstytucyjnego, in: Jan Barcz (Hrsg.): Prezyencja Niemiecka a stan debaty o reformie Unii Europejskiej, Warschau 2007, S. 57-81; und in tschechischer Sprache Stephan Hobe: Jak muze Smlouva o Ustave pro Evropu vstoupit v platnost?, in: Mezinarodni politika 1/2007, S. 34-37.

44 Mosler: Die Entstehung des Modells supranationaler und gewaltenteilender Staatenverbindungen, S. 382.

45 EuGH Rs. 294/83, Slg. 1986, S. 1339, „Les Verts“; Rs. C-314/91, Slg. 1993, S. I-1093, „Weber/Parlament“; Gutachten 1/91, Slg. 1991, S. 6079, ,EWR“; jetzt in Bezug auf den EG-Vertrag EuGH, Rs. C-15/00, Slg. 2003, S. I-7281, „Kommission/EIB““ 
Begriff der ,Verfassung ' für die Verträge zur Gründung internationaler Organisationen im Allgemeinen üblich. ${ }^{46}$ Dass der Entwurf eines Vertrages für eine europäische Verfassung diesen Begriff wieder aufgenommen hat, ist also keineswegs überraschend. Der deutliche negative Widerhall in der französischen und niederländischen Bevölkerung könnte allerdings zur Aufgabe dieser Bezeichnung führen.

Der Sache nach kann schon lange kein Zweifel mehr daran bestehen, dass Europa bereits eine Verfassung im materiellen Sinne hat. Wenn es wiederum um die Frage der supranational eigenständigen Ausübung und damit Legitimation von Hoheitsgewalt geht, so liegt es in der Konsequenz dieser Tatsache, dass es herrschaftsbegrenzender Momente europäischen Verfassungsrechts bedarf. Man wird aber darüber hinaus sogar die konkreten Verfassungsstrukturen der Herrschaft des Rechts (rule of law), der Orientierung an demokratischen Prinzipien und der Grundrechtegeltung als essenzielle Grundprinzipien europäischer Herrschaftsausübung ansehen können. Hierbei gilt es, sich von nationalstaatlich vorgeprägten Begrifflichkeiten zu lösen. ${ }^{47}$ Gewiss bedeutet zum Beispiel Demokratie auf europäischer Ebene etwas dezidiert anderes als auf nationaler Ebene. Eine entscheidende Rolle spielt dabei, dass das europäische Gesetzgebungsverfahren viel stärker exekutivisch orientiert ist als dies in jedem der Nationalstaaten mit ihren Parlamenten als ihren natürlichen Gesetzgebungsorganen der Fall sein kann. ${ }^{48}$ Legitimationsprobleme gibt es, wie oben angedeutet, inzwischen insofern tatsächlich dann, wenn die verdrängende Wirkung europäischen Sekundärrechts in das nationale Recht eine sehr bedeutende ist.

Bereits zur Zeit der Gründung der EWG war die Rolle der parlamentarischen Versammlung als demokratisches Element im Rechtssetzungsverfahren sehr schwach ausgeprägt. Im Laufe der letzten 50 Jahre hat sich das Europäische Parlament zwar eine relativ starke Vetoposition erarbeitet. ${ }^{49}$ Jedoch stellt sich immer noch die Frage, ob das bisherige Sekundärrechtsverfahren für ein ausreichendes demokratisches Fundament sorgt.

In der Tat war nun der Verfassungsvertrag durch die Stärkung des partizipativen Elements des Europäischen Parlaments am Gesetzgebungsverfahren, durch die Stärkung der Rechte der nationalen Parlamente sowie durch die Einführung des Bürgerbegehrens ein Versuch, den direkten demokratischen Einfluss und damit das Demokratieprinzip auch auf europäischer Ebene deutlich zu stärken. Insofern könnte mit der Ratifikation des Verfassungsvertrags ein weiterer bedeutender Schritt nicht nur innerhalb des Konstitutionalisierungsprozesses, sondern gerade auch für die Integration der europäischen Völker getan werden. Das Dokument setzt mit seinen vier Teilen und 436 Artikeln den supranationalen Integrationsprozess insgesamt fort, beinhaltet in diesem Sinne Ansätze für eine weitere Entwicklung der politischen Integration und verbessert die Handlungsfähigkeit der erweiterten Gemeinschaften und Union. ${ }^{50}$ Jedoch sind die Aussichten des Inkrafttretens für den Europä-

46 Mosler: Die Erweiterung des Kreises der Völkerrechtssubjekte, S. 45.

47 So wurde der Begriff des ,institutionellen Gleichgewichts“ für den traditionellen Gewaltenteilungsgrundsatz eingeführt. Zur problematischen Übertragung des nationalstaatlichen Demokratieprinzips auf die europäischen Institutionen siehe Renaud Dehousse: Beyond Representative Democracy, in: Joseph Weiler/Marlene Wind: European Constitutionalism Beyond the State, Cambridge 2003, S.135-156, hier S. 135-140.

48 Dorau: Die Verfassungsfrage der Europäischen Union, S. 86-96; Huber: Maastricht - ein Staatsstreich?, S. 3233; Winfried Kluth: Die demokratische Legitimation der Europäischen Union, Berlin 1995, S. 67-87.

49 Siehe zur Problematik des Demokratiedefizits etwa Peters: Elemente einer Theorie der Verfassung Europas, S. 626-760; Karl Doehring: Demokratiedefizit in der Europäischen Union, in: Deutsche Verwaltungsblätter 1997, S. 1133-1137; Gertrude Lübbe-Wolff: Europäisches und nationales Verfassungsrecht, in: Veröffentlichungen der Vereinigung der Deutschen Staatsrechtslehrer Heft 60, 2001, 246-287.

50 Positive Einschätzung bei Hobe: Europarecht, S. 348; Jürgen Schwarze: Ein pragmatischer Verfassungsentwurf - Analyse und Bewertung des vom Europäischen Verfassungskonvent vorgelegten Entwurfs eines Vertrags über eine Verfassung für Europa, in: Europarecht 4/2003, S. 535-573. 
ischen Verfassungsvertrag derzeit schwer zu prognostizieren. Die deutsche Ratspräsidentschaft lotet derzeit aus, ob und inwieweit Modifikationen des Verfassungsvertrages zu größerer Akzeptanz führen können. Dabei ist als erster Erfolg zu verzeichnen, dass die Diskussion über den Verfassungsvertrag beziehungsweise eine entsprechende Reform bis 2009 wieder in Gang gesetzt wurde. Bei alledem darf nicht vergessen werden, dass die Europäische Union im materiellen Sinne bereits eine Verfassung hat, und zwar gerade als Folge dieses supranationalen Integrationsprozesses seit den Römischen Verträgen.

\section{Was ist nun Europa rechtlich gesehen?}

Damit läuft unsere rechtliche ,tour d'horizon' durch die 50-jährige Entwicklung des Gemeinschaftsrechts seit den Römischen Verträgen auf die Frage hinaus, was denn nun eigentlich Europa juristisch auszeichnet. Anfänglich anvisiertes Ziel war ein europäischer Bundesstaat beziehungsweise eine europäische Föderation. Auch wenn im Sinne der DreiElemente-Lehre Georg Jellineks ${ }^{51}$ staatsähnliche Elemente auf europäischer Ebene zu finden sind, so ist doch der Staatscharakter sowohl der Gemeinschaften als auch der Union, etwa im Sinne eines Bundesstaates, abzulehnen. ${ }^{52}$ Es ist zweifelhaft, ob eine solche Staatsperspektive überhaupt Realisierung finden wird und finden soll. Andererseits - und das ist ebenso wichtig - sind die Gemeinschaften deutlich mehr als ein bloßer Staatenbund, denn die Mitgliedstaaten stehen nicht beziehungslos nebeneinander, sondern sind insbesondere durch das Recht eng miteinander verzahnt. ${ }^{53}$ Dieses Charakteristikum lässt die Typologie einer internationalen Organisation supranationalen Charakters als eher farblos erscheinen. Zu unterschiedlichen Schlussfolgerungen kommt man, wenn man einerseits die EWG und EURATOM als Staatengemeinschaften supranationalen Charakters ${ }^{54}$ und andererseits die intergouvernementalen Formen des Zusammenschlusses im Bereich der GASP und der PJZS betrachtet. Das Bundesverfassungsgericht hatte in seinem berühmten Maastricht-Urteil - damit Paul Kirchhof folgend ${ }^{55}$ - dies getan und judiziert, es handle sich beim Europa der Gemeinschaften und der Union um einen Staatenverbund. ${ }^{56}$ Damit hat das Gericht zum einen klarzustellen versucht, dass es nach wie vor verschiedene Staatsvölker in Europa gibt, und andererseits den Verbundcharakter und die rechtlich stärkere Verschränkung als in einem bloßen Staatenbund zum Ausdruck gebracht. Genau genommen ist es das Gemeinsame und Verbindende der Vielfalt und der Unterschiede in Europa, was die Gemeinschaften und die Union auszeichnet und was bereits bei der Diskussion der rechtlichen Spezifika der Integration angeklungen ist.

\section{Resümee}

Während der Vertrag zur Gründung der EURATOM im Bereich der Atomindustrie das sektorbegrenzte supranationale Integrationsmodell der Montanunion zur Erhaltung des euro-

51 Georg Jellinek: Allgemeine Staatslehre, Berlin 1914, S. 394-433; siehe dazu Dietmar Kettler: Die Drei-Elemente-Lehre - Ein Beitrag zu Jellineks Staatsbegriff, seiner Fortführung und Kritik, Münster 1995.

52 Dorau: Die Verfassungsfrage der Europäischen Union, S. 30-36; Hobe: Der offene Verfassungsstaat, S. 334379; Hobe: Der kooperationsoffene Verfassungsstaat, in: Der Staat 4/1998, S. 521-546, hier S. 539-541.

53 Dorau: Die Verfassungsfrage der Europäischen Union, S. 194-199; Jürgen Schwarze: Die Entstehung einer europäischen Verfassungsordnung, in: Jürgen Schwarze (Hrsg.): Die Entstehung einer europäischen Verfassungsordnung, Baden-Baden 2000.

54 Zum Begriff der Staatengemeinschaft siehe Hallstein: Der unvollendete Bundesstaat, S. 39-41; Mosler: Die Entstehung des Modells supranationaler und gewaltenteilender Staatenverbindungen, S. 382-383.

55 Paul Kirchhof: Der deutsche Staat im Prozess der europäischen Integration (§ 183), in: Josef Isensee/Paul Kirchhof: Handbuch des Staatsrechts der Bundesrepublik Deutschland, Heidelberg 1992, S. 879-881.

56 BVerfGE 89, 155. 
päischen Friedens übernimmt, hat insbesondere der Vertrag zur Gründung der EWG den rechtlichen Rahmen geschaffen, um auf europäischer Ebene sektorübergreifend zu regieren. Dies geschieht aber keinesfalls, ohne einen ständigen Bezug zur nationalen Ebene herzustellen, so dass von einem Mehrebenensystem gestuft organisierter öffentlicher Gewalt gesprochen werden kann. Wie bereits die Beispiele der Integration, der Supranationalität, der Grundrechte und auch der Konstitutionalisierung zeigen und dies auch durch die jeweiligen nationalen Vorschriften der Legitimation der Übertragung von Hoheitsgewalt, wie etwa dem deutschen Artikel 23 des Grundgesetzes, zum Ausdruck kommt, geht es um die Wechselbezüglichkeit und Komplementarität des nationalen und des europäischen Rechtsrahmens. Die von den Römischen Verträgen initiierten Prozesse bewirken also das gegenseitige Aufeinandereinwirken der verschiedenen rechtlichen Ebenen, welches das Bewusstsein und die Rechtswirklichkeit des Staatenverbundes bestimmt. Dies ist sein eigentlicher Motor und genau das, was die Friedenssicherungsidee der Gründerväter der Europäischen Gemeinschaften zum Inhalt hat: Weil es die Notwendigkeit der Wechselbezüglichkeit gibt, kann kein Staat in Europa mehr in diesem Staatenverbund ohne die anderen Staaten sinnvolle Entscheidungen treffen. Alle profitieren von der Kooperation und allen fehlt ein Stück Hoheitsgewalt ,zum Alleingang' durch die Tatsache, dass diese an die europäische Ebene abgegeben wurde. In diesem Sinne heißt souverän handeln zum einen das Bestimmen über die Wahl der Aufgabenerfüllungsebene, ${ }^{57}$ die in der Tat von den Mitgliedstaaten auszugehen hat, und zum anderen eine konsensorientierte Entscheidungssuche im Kreise der 26 anderen Mitgliedstaaten. Dass dies eine ganz andere, der modernen Friedenssicherungsaufgabe adäquatere Form der Ausübung von Hoheitsgewalt und damit der Souveränität ist, liegt auf der Hand. Insofern könnte man als Kategorie der ,Staats'lehre ${ }^{58}$ Wechselbezüglichkeit und Komplementarität als die neuen Grundnormen des Staatenverbundes im Sinne Hans Kelsens einführen. ${ }^{59}$ Dieses weiter zu entwickeln und noch stärker zu harmonisieren, dürfte die große Aufgabe der Zukunft sein, zu der etwa die Verabschiedung eines Europäischen Verfassungsvertrages entscheidend beitragen könnte. Dieser Verfassungsvertrag müsste dann Sinnbild eben jener Notwendigkeit der Komplementarität und Wechselbezüglichkeit sein.

Europa ist also infolge der Römischen Verträge in einem durchaus positiven Sinne zum Vorreiter einer modernen Rechtsentwicklung geworden. Dass es dies auch in der Zukunft sein kann, also mindestens in den nächsten 50 Jahren, ist nachhaltig zu hoffen und wurde auch entsprechend bei den Feierlichkeiten am 25. März 2007 mit der Berliner Erklärung60 zum Ausdruck gebracht.

57 Hobe: Der offene Verfassungsstaat, S. 390-402; dazu grundlegend auch Rainer Wahl: Der Einzelne in der Welt jenseits des Staates, in: Der Staat 1/2001, S. 45-72, hier S. 46-47, 51-57, der die globale Ebene als dritte Ebene mit einbezieht.

58 Zur notwendigen Wandlung der Kategorien der Allgemeinen Staatslehre siehe Andreas Voßkuhle: Die Renaissance der „Allgemeinen Staatlehre“ im Zeitalter der Europäisierung und Internationalisierung, in: Juristische Schulung 1/2004, S. $2-7$.

59 So ähnlich Joseph Weiler: In defence of the status quo: Europe's constitutional Sonderweg, in: Joseph Weiler/ Marlene Wind: European Constitutionalism Beyond the State, Cambridge 2003, S. 7-23, hier S. 22.

60 Abzurufen unter: http://europa.eu/50/docs/berlin_declaration_de.pdf (letzter Zugriff: 28.03.2007). 